\title{
The Effect of the Mutual Gravitational Interactions on the Perihelia Displacement of the Orbits of the Solar System's Planets
}

\author{
V.G. Vilke, A. V.Shatina, L.S. Osipova
}

The classical $N$-body problem in the case when one of the bodies (the Sun) has a much larger mass than the rest of the mutually gravitating bodies is considered. The system of equations in canonical Delaunay variables describing the motion of the system relative to the barycentric coordinate system is derived via the methods of analitical dynamics. The procedure of averaging over the fast angular variables (mean anomalies) leads to the equation describing the evolution of a single Solar system planet's perihelion as the sum of two terms. The first term corresponds to the gravitational disturbances caused by the rest of the planets, as in the case of a motionless Sun. The second appears because the problem is considered in the barycentric coordinate system and the orbits' inclinations are taken into account. This term vanishes if all planets are assumed to be moving in one static plane. This term contributes substantially to the Mercury's and Venus's perihelion evolutions. For the rest of the planet this term is small compared to the first one. For example, for Mercury the values of the two terms in question were calculated to be 528.67 and 39.64 angular seconds per century, respectively.

Keywords: N-body problem, method of averaging, Delaunay variables, orbital elements

Received May 18, 2018

Accepted July 17, 2018

Vladimir G. Vilke

polenova_t.m@mail.ru

Lyubov S. Osipova

l.s.shatina@ gmail.com

Lomonosov Moscow State University

GSP-1, Leninskie Gory, Moscow, 119991, Russia

Albina V. Shatina

shatina_av@mail.ru

Moscow Technological University (MIREA)

prosp. Vernadskogo 78, Moscow, 119454, Russia

RUSSIAN JOURNAL OF NONLINEAR DYNAMICS, 2018, 14(3), 291-300 


\section{Formulation of the problem. Equations of motion}

Let us consider a model of the Solar system, consisting of $(n+1)$ material points $P_{0}, P_{1}, \ldots, P_{n}$. Let us introduce an inertial coordinate system $C X Y Z$ with its origin at the system's center of mass. Let $\mathbf{R}_{0}$ be the radius vector of point $P_{0}$ (the Sun), and let $\mathbf{R}_{1}, \ldots, \mathbf{R}_{n}$ be the radius vectors of planets $P_{1}, \ldots, P_{n}$, whose coordinates are written in the coordinate system $C X Y Z$.

Let us denote the mass of the Sun as $m_{s}$ and the masses of the planets as $M_{k}(k=1, \ldots, n)$. Introducing the small parameter $\varepsilon$, equal to the ratio of the mass of the heaviest planet (Jupiter) to the mass of the Sun, $\varepsilon=M_{5} m_{s}^{-1}$, we can represent the masses of other planets as $M_{k}=\varepsilon m_{k}$, $k=1, \ldots, n$, where $m_{k}=M_{k} M_{5}^{-1} m_{s}, m_{k} \leqslant m_{s}$.

Since the origin of the inertial coordinate system coincides with the barycenter,

$$
m_{s} \mathbf{R}_{0}+\varepsilon \sum_{k=1}^{n} m_{k} \mathbf{R}_{k}=0
$$

The kinetic energy equals

$$
T=\frac{1}{2} m_{s} \dot{\mathbf{R}}_{0}^{2}+\frac{1}{2} \sum_{k=1}^{n} \varepsilon m_{k} \dot{\mathbf{R}}_{k}^{2}
$$

Considering (1.1) and (1.2), we can obtain that

$$
T=\frac{\varepsilon}{2} \sum_{k=1}^{n} m_{k} \dot{\mathbf{R}}_{k}^{2}+\frac{\varepsilon^{2}}{2 m_{s}}\left(\sum_{k=1}^{n} m_{k} \dot{\mathbf{R}}_{k}\right)^{2} .
$$

The potential energy of gravitational forces equals

$$
\Pi=-\sum_{k=1}^{n} \frac{\varepsilon f_{0} m_{k}}{\left[\left(\mathbf{R}_{0}-\mathbf{R}_{k}\right)^{2}\right]^{1 / 2}}-\sum_{\substack{i, j=1, i<j}}^{n} \frac{\varepsilon^{2} f m_{i} m_{j}}{R_{i j}},
$$

where $f$ is the universal gravitational constant, $f_{0}=f m_{s}, R_{i j}=\left|\mathbf{R}_{i}-\mathbf{R}_{j}\right|$.

Considering equality (1.1) and keeping terms of no higher than first order by $\varepsilon$, we can rewrite the expression (1.4) as

$$
\Pi=-\varepsilon \sum_{k=1}^{n} \frac{f_{0} m_{k}}{R_{k}}-\varepsilon^{2} \sum_{\substack{i, j=1, i<j}}^{n} \frac{f m_{i} m_{j}}{R_{i j}}+\varepsilon^{2} \sum_{i, k=1}^{n} \frac{f m_{i} m_{k}\left(\mathbf{R}_{i}, \mathbf{R}_{k}\right)}{R_{k}^{3}},
$$

where $R_{k}=\left|\mathbf{R}_{k}\right|$.

The Lagrangian function of the mechanical system under study is defined as

$$
L=T-\Pi \text {. }
$$

Let us write the Lagrangian function as

$$
L=\varepsilon L_{1}+\varepsilon^{2} L_{2},
$$


where

$$
\begin{aligned}
& L_{1}=\frac{1}{2} \sum_{k=1}^{n} m_{k} \dot{\mathbf{R}}_{k}^{2}+\sum_{k=1}^{n} \frac{f_{0} m_{k}}{R_{k}} \\
& L_{2}=\frac{1}{2 m_{s}}\left(\sum_{k=1}^{n} m_{k} \dot{\mathbf{R}}_{k}\right)^{2}+\sum_{\substack{i, j=1, i<j}}^{n} \frac{f m_{i} m_{j}}{R_{i j}}-\sum_{i, k=1}^{n} \frac{f m_{i} m_{k}\left(\mathbf{R}_{i}, \mathbf{R}_{k}\right)}{R_{k}^{3}} .
\end{aligned}
$$

Function $L_{1}$ and the corresponding equations describe the Kepler motions of each planet in the central force field along an elliptic trajectory. The function $L_{2}$ defines the perturbations of the Kepler motions.

Let us consider the problem of perturbed motion of the planet with the following Lagrangian function:

$$
\widetilde{L}=L_{1}+\varepsilon L_{2}
$$

We shall use the Delaunay canonical variables [1] to describe the motion of the planets. The first step is to transform from generalized coordinates and velocities $\left(\mathbf{R}_{k}, \dot{\mathbf{R}}_{k}\right)$ to generalized coordinates and impulses $\left(\mathbf{R}_{k}, \mathbf{p}_{k}\right), k=1, \ldots, n$ :

$$
\mathbf{p}_{k}=\nabla_{\dot{\mathbf{R}}_{k}} \widetilde{L}=m_{k} \dot{\mathbf{R}}_{k}+\frac{\varepsilon m_{k}}{m_{s}} \sum_{i=1}^{n} m_{i} \dot{\mathbf{R}}_{i}, \quad k=1, \ldots, n .
$$

Let $\mathbf{p}=\left(\mathbf{p}_{1}, \ldots, \mathbf{p}_{n}\right), \mathbf{R}=\left(\mathbf{R}_{1}, \ldots, \mathbf{R}_{n}\right)$. Then equality (1.9) can be written as

$$
\mathbf{p}=\Lambda_{1}\left(E+\varepsilon \Lambda_{2}\right) \dot{\mathbf{R}}
$$

where

$$
\begin{gathered}
\Lambda_{1}=\operatorname{diag}\left\{m_{1}, \ldots, m_{n}\right\}, \quad \Lambda_{2}=\left(\frac{\delta_{i i} m_{j}}{m_{s}}\right)_{i, j=1}^{n}, \\
E=\left(\delta_{i j}\right)_{i, j=1}^{n}, \quad \delta_{i j}=\left\{\begin{array}{l}
1, i=j, \\
0, i \neq j .
\end{array}\right.
\end{gathered}
$$

Keeping terms of no higher than first order by $\varepsilon$, we can obtain from (1.10) that

$$
\begin{gathered}
\dot{\mathbf{R}}=\left[\Lambda_{1}\left(E+\varepsilon \Lambda_{2}\right)\right]^{-1} \mathbf{p} \approx\left(E-\varepsilon \Lambda_{2}\right) \Lambda_{1}^{-1} \mathbf{p}=\Lambda_{1}^{-1} \mathbf{p}-\varepsilon \Lambda_{2} \Lambda_{1}^{-1} \mathbf{p}, \\
\Lambda_{1}^{-1}=\operatorname{diag}\left\{\frac{1}{m_{1}}, \ldots, \frac{1}{m_{n}}\right\}, \quad \Lambda_{2} \Lambda_{1}^{-1}=\left(\frac{\delta_{i i} \delta_{j j}}{m_{s}}\right)_{i, j=1}^{n} .
\end{gathered}
$$

Therefore,

$$
\dot{\mathbf{R}}_{k}=\frac{1}{m_{k}} \mathbf{p}_{k}-\frac{\varepsilon}{m_{s}} \sum_{i=1}^{n} \mathbf{p}_{i}, \quad k=1, \ldots, n .
$$

The Hamiltonian function is defined by the following equality [2]:

$$
\mathcal{H}=\sum_{k=1}^{n}\left(\mathbf{p}_{k}, \dot{\mathbf{R}}_{k}\right)-\left.\widetilde{L}\right|_{\dot{\mathbf{R}} \rightarrow \mathbf{p}} .
$$


Considering (1.6)-(1.8) and (1.11), we can obtain

$$
\begin{gathered}
\mathcal{H}=\mathcal{H}_{0}+\varepsilon \mathcal{H}_{1}, \\
\mathcal{H}_{0}=\sum_{k=1}^{n} \frac{\mathbf{p}_{k}^{2}}{2 m_{k}}-\sum_{k=1}^{n} \frac{f_{0} m_{k}}{R_{k}}, \\
\mathcal{H}_{1}=-\frac{1}{2 m_{s}} \sum_{k, i=1}^{n}\left(\mathbf{p}_{k}, \mathbf{p}_{i}\right)-\sum_{\substack{i, j=1, i<j}}^{n} \frac{f m_{i} m_{j}}{R_{i j}}+\sum_{k, i=1}^{n} \frac{f m_{i} m_{k}\left(\mathbf{R}_{k}, \mathbf{R}_{i}\right)}{R_{k}^{3}} .
\end{gathered}
$$

At our next step we shall use spherical coordinates $R_{k}, \varphi_{k}, \psi_{k}$ of vectors $\mathbf{R}_{k}(k=1, \ldots, n)$ :

$$
\mathbf{R}_{k}=R_{k} \boldsymbol{\xi}_{k}, \boldsymbol{\xi}_{k}=\left(\cos \varphi_{k} \sin \psi_{k}, \sin \varphi_{k} \sin \psi_{k}, \cos \psi_{k}\right)
$$

Then

$$
\dot{\mathbf{R}}_{k}=\dot{R}_{k} \boldsymbol{\xi}_{k}+R_{k} \dot{\varphi}_{k} \boldsymbol{\eta}_{k}+R_{k} \dot{\psi}_{k} \boldsymbol{\zeta}_{k}
$$

where

$$
\begin{aligned}
& \boldsymbol{\eta}_{k}=\left(-\sin \varphi_{k} \sin \psi_{k}, \cos \varphi_{k} \sin \psi_{k}, 0\right), \\
& \boldsymbol{\zeta}_{k}=\left(\cos \varphi_{k} \cos \psi_{k}, \sin \varphi_{k} \cos \psi_{k},-\sin \psi_{k}\right)
\end{aligned}
$$

Let us express $\mathbf{p}_{k}$ in terms of generalized impulses $p_{R_{k}}, p_{\varphi_{k}}, p_{\psi_{k}}$, corresponding to generalized coordinates $R_{k}, \varphi_{k}, \psi_{k}(k=1, \ldots, n)$ :

$$
\begin{aligned}
& p_{R_{k}}=\frac{\partial \widetilde{L}}{\partial \dot{R}_{k}}=\left(\nabla_{\dot{\mathbf{R}}_{k}} \widetilde{L}, \frac{\partial \dot{\mathbf{R}}_{k}}{\partial \dot{R}_{k}}\right)=\left(\mathbf{p}_{k}, \boldsymbol{\xi}_{k}\right), \\
& p_{\varphi_{k}}=\frac{\partial \widetilde{L}}{\partial \dot{\varphi}_{k}}=\left(\nabla_{\dot{\mathbf{R}}_{k}} \widetilde{L}, \frac{\partial \dot{\mathbf{R}}_{k}}{\partial \dot{\varphi}_{k}}\right)=\left(\mathbf{p}_{k}, R_{k} \boldsymbol{\eta}_{k}\right), \\
& p_{\psi_{k}}=\frac{\partial \widetilde{L}}{\partial \dot{\psi}_{k}}=\left(\nabla_{\dot{\mathbf{R}}_{k}} \widetilde{L}, \frac{\partial \dot{\mathbf{R}}_{k}}{\partial \dot{\psi}_{k}}\right)=\left(\mathbf{p}_{k}, R_{k} \boldsymbol{\zeta}_{k}\right) .
\end{aligned}
$$

Then from (1.14)-(1.18) we get

$$
\mathbf{p}_{k}=p_{R_{k}} \boldsymbol{\xi}_{k}+\frac{p_{\varphi_{k}}}{R_{k} \sin ^{2} \psi_{k}} \boldsymbol{\eta}_{k}+\frac{p_{\psi_{k}}}{R_{k}} \zeta_{k} .
$$

Let us transform from generalized impulses and coordinates $p_{R_{k}}, p_{\varphi_{k}}, p_{\psi_{k}}, R_{k}, \varphi_{k}, \psi_{k}$ to Delaunay variables $L_{k}, G_{k}, H_{k}, l_{k}, g_{k}, h_{k}(k=1, \ldots, n)$ using the generating function of canonical transformation

$$
\begin{aligned}
W & =\sum_{k=1}^{n} W_{k}, \\
W_{k} & =W_{k}\left[R_{k}, \varphi_{k}, \psi_{k}, L_{k}, G_{k}, H_{k}\right] \\
& =H_{k} \varphi_{k}+\int \sqrt{G_{k}^{2}-\frac{H_{k}^{2}}{\sin ^{2} \psi_{k}}} d \psi_{k}+\int \sqrt{\frac{2 f_{0} m_{k}^{2}}{R_{k}}-\frac{G_{k}^{2}}{R_{k}^{2}}-\frac{f_{0}^{2} m_{k}^{4}}{L_{k}^{2}}} d R_{k} .
\end{aligned}
$$



form:

The system of equations defining the transformation to Delaunay variables has the following

$$
\begin{aligned}
p_{R_{k}} & =\frac{\partial W}{\partial R_{k}}, & p_{\varphi_{k}} & =\frac{\partial W}{\partial \varphi_{k}}, \\
l_{k} & =\frac{\partial W}{\partial L_{k}}, & p_{\psi_{k}} & =\frac{\partial W}{\partial \psi_{k}}, \\
g_{k} & =\frac{\partial W}{\partial G_{k}}, & h_{k} & =\frac{\partial W}{\partial H_{k}} .
\end{aligned}
$$

The magnitude of $\mathbf{R}_{k}$ can be expressed in terms of Delaunay variables as

$$
R_{k}=\frac{G_{k}^{2}}{f_{0} m_{k}^{2} \lambda_{k}}
$$

where $\lambda_{k}=1+e_{k} \cos \vartheta_{k}, e_{k}=\sqrt{1-\frac{G_{k}^{2}}{L_{k}^{2}}}$ is the eccentricity of the $k$ th planet's orbit, and $\vartheta_{k}=\vartheta_{k}\left(l_{k}, L_{k}, G_{k}\right)$ is the true anomaly defined by $l_{k}, L_{k}, G_{k}$ via the relations

$$
\cos w_{k}=\frac{e_{k}+\cos \vartheta_{k}}{1+e_{k} \cos \vartheta_{k}}, \quad l_{k}=w_{k}-e_{k} \sin w_{k}
$$

The unit vectors $\boldsymbol{\xi}_{k}$ in the inertial coordinate system can be expressed in Delaunay variables as

$$
\begin{gathered}
\boldsymbol{\xi}_{k}=\left(\xi_{k x}, \xi_{k y}, \xi_{k z}\right), \\
\xi_{k x}=\cos \left(g_{k}+\vartheta_{k}\right) \cos h_{k}-\sin \left(g_{k}+\vartheta_{k}\right) \cos i_{k} \sin h_{k}, \\
\xi_{k y}=\cos \left(g_{k}+\vartheta_{k}\right) \sin h_{k}+\sin \left(g_{k}+\vartheta_{k}\right) \cos i_{k} \cos h_{k}, \\
\xi_{k z}=\sin \left(g_{k}+\vartheta_{k}\right) \sin i_{k},
\end{gathered}
$$

where $i_{k}$ is the $k$ th planet's orbit inclination, $\cos i_{k}=H_{k} / G_{k}, g_{k}$ is the longitude of the perihelion from the ascending node, and $h_{k}$ is the longitude of the ascending node.

From relations (1.14), (1.16) and (1.23) we can obtain expressions for the coordinates of the vectors $\boldsymbol{\eta}_{k}$ in terms of Delaunay variables:

$$
\boldsymbol{\eta}_{k}=\left(-\xi_{k y}, \xi_{k x}, 0\right) \text {. }
$$

The components of $\boldsymbol{\zeta}_{k}$ are defined by the following relations:

$$
\left(\boldsymbol{\zeta}_{k}, \boldsymbol{\xi}_{k}\right)=0, \quad\left(\boldsymbol{\zeta}_{k}, \boldsymbol{\eta}_{k}\right)=0, \quad\left(\boldsymbol{\zeta}_{k}, \boldsymbol{\zeta}_{k}\right)=1, \quad \zeta_{k z} \leqslant 0 .
$$

Thus, it follows from (1.23)-(1.25) that

$$
\zeta_{k}=\left(\frac{\xi_{k x} \xi_{k z}}{\sqrt{1-\xi_{k z}^{2}}}, \frac{\xi_{k y} \xi_{k z}}{\sqrt{1-\xi_{k z}^{2}}},-\sqrt{1-\xi_{k z}^{2}}\right)
$$

The generalized impulses $p_{R_{k}}, p_{\varphi_{k}}, p_{\psi_{k}}$ can be expressed through Delaunay variables from $(1.20)-(1.22)$ as

$$
\begin{aligned}
& p_{R_{k}}=\frac{f_{0} m_{k}^{2}}{G_{k}} e_{k} \sin \vartheta_{k}, \quad p_{\varphi_{k}}=H_{k} \\
& p_{\psi_{k}}=\frac{G_{k} \sin i_{k} \cos \left(g_{k}+\vartheta_{k}\right)}{\sqrt{1-\sin ^{2}\left(g_{k}+\vartheta_{k}\right) \sin ^{2} i_{k}}} .
\end{aligned}
$$


Using (1.19), (1.23), (1.24), (1.26) and (1.27), we get

$$
\begin{gathered}
\mathbf{p}_{k}=D_{k}\left\{e_{k} \sin \vartheta_{k} \boldsymbol{\xi}_{k}+\frac{\lambda_{k} \cos i_{k}}{1-\sin ^{2}\left(g_{k}+\vartheta_{k}\right) \sin ^{2} i_{k}} \boldsymbol{\eta}_{k}+\frac{\lambda_{k} \sin i_{k} \cos \left(g_{k}+\vartheta_{k}\right)}{\sqrt{1-\sin ^{2}\left(g_{k}+\vartheta_{k}\right) \sin ^{2} i_{k}}} \boldsymbol{\zeta}_{k}\right\}, \\
D_{k}=\frac{f_{0} m_{k}^{2}}{G_{k}} .
\end{gathered}
$$

The unperturbed part of the Hamiltonian (1.12) in terms of Delaunay variables has the following form:

$$
\mathcal{H}_{0}=-\sum_{k=1}^{n} \frac{f_{0}^{2} m_{k}^{3}}{2 L_{k}^{2}}
$$

The perturbed part of the Hamiltonian (1.12) is defined by (1.13), where vectors $\mathbf{p}_{k}$ are expressed in terms of Delaunay variables according to (1.28), and $\mathbf{R}_{k}=R_{k} \boldsymbol{\xi}_{k}$ according to $(1.22)-(1.23)$.

The canonical equations of motion have the following form:

$$
\begin{aligned}
& \dot{L}_{k}=-\varepsilon \frac{\partial \mathcal{H}_{1}}{\partial l_{k}}, \dot{l}_{k}=\omega_{k}+\varepsilon \frac{\partial \mathcal{H}_{1}}{\partial L_{k}}, \quad \omega_{k}=\frac{f_{0}^{2} m_{k}^{3}}{L_{k}^{3}}, \\
& \dot{G}_{k}=-\varepsilon \frac{\partial \mathcal{H}_{1}}{\partial g_{k}}, \quad \dot{g}_{k}=\varepsilon \frac{\partial \mathcal{H}_{1}}{\partial G_{k}}, \\
& \dot{H}_{k}=-\varepsilon \frac{\partial \mathcal{H}_{1}}{\partial h_{k}}, \quad \dot{h}_{k}=\varepsilon \frac{\partial \mathcal{H}_{1}}{\partial H_{k}}, \quad k=1, \ldots, n .
\end{aligned}
$$

The right-hand sides of Eqs. (1.30) depend on canonical Delaunay variables and contain $n$ fast angular variables $l_{1}, \ldots, l_{n}$, by which the function $\mathcal{H}_{1}$ is $2 \pi$-periodic. The rest of the variables are slow. Let us assume that there are no resonances in the system, i.e., $k_{1} \omega_{1}+\ldots+k_{n} \omega_{n} \neq 0$ for any nontrivial set of integer numbers $k_{1}, \ldots, k_{n}$, and apply the method of averaging by fast variables. The operation of averaging consists in calculating the following integral:

$$
\langle(*)\rangle_{l}=\frac{1}{(2 \pi)^{n}} \int_{0}^{2 \pi} \ldots \int_{0}^{2 \pi}(*) d l_{1} \ldots d l_{n}=\frac{1}{(2 \pi)^{n}} \int_{0}^{2 \pi} \ldots \int_{0}^{2 \pi}(*) \frac{\partial l_{1}}{\partial \vartheta_{1}} \ldots \frac{\partial l_{n}}{\partial \vartheta_{n}} d \vartheta_{1} \ldots d \vartheta_{n},
$$

where $\frac{\partial l_{k}}{\partial \vartheta_{k}}=\frac{\left(1-e_{k}^{2}\right)^{3 / 2}}{\lambda_{k}^{2}}, k=1, \ldots, n$.

\section{The perihelion longitude evolution of the $j$ th planet}

Let us derive the equation describing the evolution of the perihelion longitude for the $j$ th planet, $\varpi_{1}=g_{1}+h_{1}$. According to (1.30),

$$
\left\langle\dot{\varpi}_{j}\right\rangle=\varepsilon\left\langle\frac{\partial \mathcal{H}_{1}}{\partial G_{j}}+\frac{\partial \mathcal{H}_{1}}{\partial H_{j}}\right\rangle
$$


Taking into consideration only those summands of $\mathcal{H}_{1}$ that depend on $G_{j}$ and $H_{j}$, we obtain

$$
\begin{aligned}
\left\langle\dot{\varpi}_{j}\right\rangle & =\varepsilon\left\langle( \frac { \partial } { \partial G _ { j } } + \frac { \partial } { \partial H _ { j } } ) \left(-\frac{1}{2 m_{s}} \mathbf{p}_{j}^{2}+\frac{f m_{j}^{2}}{R_{j}}-\frac{1}{m_{s}} \sum_{\substack{k=1, k \neq j}}^{n}\left(\mathbf{p}_{j}, \mathbf{p}_{k}\right)-\right.\right. \\
& \left.\left.-\sum_{\substack{k=1, k \neq j}}^{n} \frac{f m_{j} m_{k}}{R_{j k}}+\sum_{\substack{k=1, k \neq j}}^{n} f m_{j} m_{k}\left(\mathbf{R}_{j}, \mathbf{R}_{k}\right)\left(\frac{1}{R_{j}^{3}}+\frac{1}{R_{k}^{3}}\right)\right)\right\rangle .
\end{aligned}
$$

Let us note that

$$
-\frac{1}{2 m_{s}} \mathbf{p}_{j}^{2}+\frac{f m_{j}^{2}}{R_{j}}=\frac{f_{0}^{2} m_{j}^{4}}{2 m_{s} L_{j}^{2}} .
$$

Therefore, the partial derivatives of this expression by $G_{j}$ and $H_{j}$ are equal to zero. Next,

$$
\left\langle\frac{\mathbf{R}_{k}}{R_{k}^{3}}\right\rangle=\frac{\left(1-e_{k}^{2}\right)^{3 / 2}}{2 \pi} \int_{0}^{2 \pi} \frac{\boldsymbol{\xi}_{k} d \vartheta_{k}}{\lambda_{k}^{2} R_{k}^{2}}=\frac{\left(1-e_{k}^{2}\right)^{3 / 2}}{2 \pi} \frac{f_{0}^{2} m_{k}^{4}}{G_{k}^{4}} \int_{0}^{2 \pi} \boldsymbol{\xi}_{k} d \vartheta_{k}=0 .
$$

After accounting for all the relations above, relation (2.1) takes the following form:

$$
\left\langle\dot{\varpi}_{j}\right\rangle=\sum_{\substack{k=1, k \neq j}}^{n} \omega_{j k}^{(p)}+\sum_{\substack{k=1, k \neq j}}^{n} \omega_{j k}^{(R)}
$$

where

$$
\begin{aligned}
& \omega_{j k}^{(p)}=-\frac{\varepsilon}{m_{s}}\left\langle\left(\frac{\partial}{\partial G_{j}}+\frac{\partial}{\partial H_{j}}\right)\left(\mathbf{p}_{j}, \mathbf{p}_{k}\right)\right\rangle, \\
& \omega_{j k}^{(R)}=-\varepsilon\left\langle\left(\frac{\partial}{\partial G_{j}}+\frac{\partial}{\partial H_{j}}\right) \frac{f m_{j} m_{k}}{R_{j k}}\right\rangle .
\end{aligned}
$$

The coordinates of the impulse vector $\mathbf{p}_{k}(1.28)$ can be represented as

$$
\begin{aligned}
& p_{k x}=D_{k}\left[M_{k} \cos h_{k}-N_{k} \sin h_{k}\right], \\
& p_{k y}=D_{k}\left[N_{k} \cos h_{k}+M_{k} \sin h_{k}\right], \quad p_{k z}=D_{k} Q_{k},
\end{aligned}
$$

where

$$
\begin{aligned}
M_{k} & =M_{k}\left(e_{k}, g_{k}, i_{k}, \vartheta_{k}\right)=e_{k} \sin \vartheta_{k} \cos \left(g_{k}+\vartheta_{k}\right)+ \\
& +\frac{\lambda_{k} \sin \left(g_{k}+\vartheta_{k}\right)\left[\sin ^{2} i_{k} \cos ^{2}\left(g_{k}+\vartheta_{k}\right)-\cos ^{2} i_{k}\right]}{1-\sin ^{2}\left(g_{k}+\vartheta_{k}\right) \sin ^{2} i_{k}}, \\
N_{k} & =N_{k}\left(e_{k}, g_{k}, i_{k}, \vartheta_{k}\right)=\left[e_{k} \sin \vartheta_{k} \sin \left(g_{k}+\vartheta_{k}\right)+\right. \\
& \left.+\frac{\lambda_{k} \cos \left(g_{k}+\vartheta_{k}\right)\left[1+\sin ^{2} i_{k} \sin ^{2}\left(g_{k}+\vartheta_{k}\right)\right]}{1-\sin ^{2}\left(g_{k}+\vartheta_{k}\right) \sin ^{2} i_{k}}\right] \cos i_{k}, \\
Q_{k} & =Q_{k}\left(e_{k}, g_{k}, i_{k}, \vartheta_{k}\right)=\left[e_{k} \sin \vartheta_{k} \sin \left(g_{k}+\vartheta_{k}\right)-\lambda_{k} \cos \left(g_{k}+\vartheta_{k}\right)\right] \sin i_{k} .
\end{aligned}
$$


Let us denote

$$
\begin{aligned}
\widetilde{M}_{k} & =\widetilde{M}_{k}\left(e_{k}, g_{k}, i_{k}\right)=\left\langle M_{k}\left(e_{k}, g_{k}, i_{k}, \vartheta_{k}\right)\right\rangle, \\
\widetilde{N}_{k} & =\widetilde{N}_{k}\left(e_{k}, g_{k}, i_{k}\right)=\left\langle N_{k}\left(e_{k}, g_{k}, i_{k}, \vartheta_{k}\right)\right\rangle, \\
\widetilde{Q}_{k} & =\widetilde{Q}_{k}\left(e_{k}, g_{k}, i_{k}\right)=\left\langle Q_{k}\left(e_{k}, g_{k}, i_{k}, \vartheta_{k}\right)\right\rangle .
\end{aligned}
$$

Then

$$
\begin{gathered}
\left\langle\left(\mathbf{p}_{j}, \mathbf{p}_{k}\right)\right\rangle=\frac{f_{0}^{2} m_{j}^{2} m_{k}^{2}\left(1-e_{j}^{2}\right)^{3 / 2}\left(1-e_{k}^{2}\right)^{3 / 2}}{G_{j} G_{k}} F_{j k}, \\
F_{j k}=\left(\widetilde{M}_{j} \widetilde{M}_{k}+\widetilde{N}_{j} \widetilde{N}_{k}\right) \cos \left(h_{j}-h_{k}\right)+\left(\widetilde{M_{j}} \widetilde{N}_{k}-\widetilde{N}_{j} \widetilde{M}_{k}\right) \sin \left(h_{j}-h_{k}\right)+\widetilde{Q}_{j} \widetilde{Q}_{k} .
\end{gathered}
$$

In view of the relations

$$
\begin{gathered}
\frac{\partial}{\partial G_{k}} \frac{\left(1-e_{k}^{2}\right)^{3 / 2}}{G_{k}}=\frac{2}{G_{k}^{2}}\left(1-e_{k}^{2}\right)^{3 / 2}, \quad \frac{\partial e_{k}}{\partial G_{k}}=\frac{e_{k}^{2}-1}{e_{k} G_{k}}, \quad \frac{\partial i_{k}}{\partial G_{k}}+\frac{\partial i_{k}}{\partial H_{k}}=\frac{\cos i_{k}-1}{G_{k} \sin i_{k}}, \\
G_{k}=\sqrt{f_{0} m_{k}^{2} a_{k}\left(1-e_{k}^{2}\right)}, \quad k=1, \ldots, n,
\end{gathered}
$$

where $a_{k}$ is the semimajor axis of the orbit of the $k$ th planet, the summands $\omega_{j k}^{(p)}$ in $(2.2)$ can be expressed as

$$
\omega_{j k}^{(p)}=-\frac{\varepsilon m_{k}\left(1-e_{k}^{2}\right)}{a_{j}} \sqrt{\frac{f\left(1-e_{j}^{2}\right)}{m_{s} a_{k}}}\left\{2 F_{j k}+\frac{\partial F_{j k}}{\partial e_{j}} \cdot \frac{e_{j}^{2}-1}{e_{j}}+\frac{\partial F_{j k}}{\partial i_{j}} \cdot \frac{\cos i_{j}-1}{\sin i_{j}}\right\} .
$$

Let us express $\omega_{j k}^{(R)}$ in (2.2) in terms of Delaunay variables. Since

$$
R_{j k}=\sqrt{\left(\mathbf{R}_{j}-\mathbf{R}_{k}\right)^{2}}=\sqrt{R_{j}^{2}-2 R_{j} R_{k}\left(\boldsymbol{\xi}_{j}, \boldsymbol{\xi}_{k}\right)+R_{k}^{2}},
$$

we have

$$
\begin{aligned}
\left(\frac{\partial}{\partial G_{j}}+\frac{\partial}{\partial H_{j}}\right) \frac{1}{R_{j k}} & =-\frac{1}{\left(R_{j}^{2}-2 R_{j} R_{k}\left(\boldsymbol{\xi}_{j}, \boldsymbol{\xi}_{k}\right)+R_{k}^{2}\right)^{3 / 2}} \times \\
& \times\left\{R_{j} \frac{\partial R_{j}}{\partial G_{j}}-\frac{\partial R_{j}}{\partial G_{j}} R_{k}\left(\boldsymbol{\xi}_{j}, \boldsymbol{\xi}_{k}\right)-R_{j} R_{k}\left(\frac{\partial \boldsymbol{\xi}_{j}}{\partial G_{j}}+\frac{\partial \boldsymbol{\xi}_{j}}{\partial H_{j}}, \boldsymbol{\xi}_{k}\right)\right\} .
\end{aligned}
$$

Let us modify the right-hand side of (2.4) using equality (1.22) for $R_{j}, R_{k}$ and the following expressions for the partial derivatives:

$$
\begin{gathered}
\frac{\partial R_{j}}{\partial G_{j}}=\frac{G_{j} \cos \vartheta_{j}}{f_{0} m_{j}^{2} e_{j}}, \quad \frac{\partial \vartheta_{j}}{\partial G_{j}}=-\frac{\sin \vartheta_{j}\left(2+e_{j} \cos \vartheta_{j}\right)}{e_{j} G_{j}} \\
\frac{\partial \boldsymbol{\xi}_{j}}{\partial G_{j}}+\frac{\partial \boldsymbol{\xi}_{j}}{\partial H_{j}}=\frac{\partial \boldsymbol{\xi}_{j}}{\partial \vartheta_{j}} \frac{\partial \vartheta_{j}}{\partial G_{j}}+\frac{\partial \boldsymbol{\xi}_{j}}{\partial i_{j}}\left(\frac{\partial i_{j}}{\partial G_{j}}+\frac{\partial i_{j}}{\partial H_{j}}\right) .
\end{gathered}
$$


In the end we get

$$
\omega_{j k}^{(R)}=\frac{\varepsilon m_{k}\left(1-e_{j}^{2}\right)^{2}}{4 \pi^{2} e_{j} a_{k}^{2}} \sqrt{\frac{f a_{j}}{m_{s}\left(1-e_{k}^{2}\right)}} \int_{0}^{2 \pi} \int_{0}^{2 \pi} \Phi_{j k} d \vartheta_{j} d \vartheta_{k},
$$

where

$$
\begin{gathered}
\Phi_{j k}=\left\{\lambda_{j}^{2}-2 \mu_{j k} \lambda_{j} \lambda_{k}\left(\boldsymbol{\xi}_{j}, \boldsymbol{\xi}_{k}\right)+\lambda_{k}^{2} \mu_{1 k}^{2}\right\}^{-3 / 2} \times \\
\times\left\{\mu_{j k} \lambda_{k} \cos \vartheta_{j}-\lambda_{j} \cos \vartheta_{j}\left(\boldsymbol{\xi}_{j}, \boldsymbol{\xi}_{k}\right)+\left(\frac{\partial \boldsymbol{\xi}_{j}}{\partial \vartheta_{j}}, \boldsymbol{\xi}_{k}\right) \sin \vartheta_{j}\left(1+\lambda_{j}\right)+\left(\frac{\partial \boldsymbol{\xi}_{j}}{\partial i_{j}}, \boldsymbol{\xi}_{k}\right) \frac{\left(1-\cos i_{j}\right) e_{j}}{\sin i_{j}}\right\}, \\
\mu_{j k}=\frac{G_{j}^{2} m_{k}^{2}}{G_{k}^{2} m_{j}^{2}}=\frac{a_{j}\left(1-e_{j}^{2}\right)}{a_{k}\left(1-e_{k}^{2}\right)} .
\end{gathered}
$$

Let us find the numerical values of components $\omega_{j k}^{(R)}, \omega_{j k}^{(p)}$ for each of the eight planets in the Solar System for the epoch J2000, using the orbital elements given in [3] and the formulas (2.3) and (2.5). The results of these calculations are presented in Tables 1 and 2. All the calculations are performed using the mathematical system Mathcad.

The term $\omega_{j}^{R}=\sum_{k=1, k \neq j}^{n} \omega_{j k}^{(R)}$ is responsible for the gravitational disturbances caused by the rest of the planet, as in the case of the motionless Sun. The values obtained match the results of calculations performed by U. Le Verrier in 1859 [4] for Mercury and by G. M. Clemence [5, 6] for Mercury and Earth.

The component $\omega_{j}^{(p)}=\sum_{k=1, k \neq j}^{n} \omega_{j k}^{(p)}$ has appeared because the problem is considered in the barycentric coordinate system and the orbit inclinations are taken into account. This component becomes zero under the assumption that all the planets move in one static plane. The term $\omega_{j}^{(p)}$ influences the Mercury's and Venus's evolution of the perihelion considerably. For the rest of the planets this term is small relative to $\omega_{j}^{(R)}$.

Table 1. The evolution of orbits' perihelia, component $\omega_{j}^{(R)}$

\begin{tabular}{|l|c|c|c|c|c|c|c|c|}
\hline \multirow{2}{*}{$\begin{array}{c}\text { Influencing } \\
\text { planets }\end{array}$} & \multicolumn{7}{|c|}{ Influenced planets } \\
\cline { 2 - 10 } & Mercury & Venus & Earth-Moon & Mars & Jupiter & Saturn & Uranus & Neptune \\
\hline Mercury & - & -150.94 & -13.71 & 0.77 & 0.01 & 0.001 & 0.000 & 0.00 \\
\hline Venus & 275.89 & - & 345.15 & 49.40 & 0.41 & 0.05 & 0.004 & 0.001 \\
\hline Earth-Moon & 90.10 & -574.49 & - & 227.61 & 0.99 & 0.11 & 0.01 & 0.002 \\
\hline Mars & 2.46 & 74.88 & 97.45 & - & 0.12 & 0.03 & 0.003 & 0.00 \\
\hline Jupiter & 152.77 & 652.78 & 694.13 & 1247.15 & - & 1626.28 & 124.72 & -0.86 \\
\hline Saturn & 7.27 & 8.18 & 19.19 & 66.67 & 628.38 & - & 121.16 & -13.82 \\
\hline Uranus & 0.14 & 0.28 & 0.56 & 1.20 & 10.74 & 27.03 & - & 97.29 \\
\hline Neptune & 0.04 & 0.11 & 0.18 & 0.35 & 2.24 & 6.33 & 45.24 & - \\
\hline$\omega_{j}^{(R)}$ & 528.67 & 10.8 & 1142.95 & 1593.15 & 642.89 & 1659.83 & 291.14 & 82.61 \\
\hline
\end{tabular}


Table 2. The evolution of orbits' perihelia, component $\omega_{j}^{(p)}$

\begin{tabular}{|l|c|c|c|c|c|c|c|c|}
\hline \multirow{2}{*}{$\begin{array}{c}\text { Influencing } \\
\text { planets }\end{array}$} & \multicolumn{7}{|c|}{ Influenced planets } \\
\cline { 2 - 10 } & Mercury & Venus & Earth-Moon & Mars & Jupiter & Saturn & Uranus & Neptune \\
\hline Mercury & - & 5.22 & -0.00 & 0.05 & 0.005 & 0.06 & -0.001 & 0.01 \\
\hline Venus & 0.11 & - & -0.00 & 0.01 & 0.001 & 0.01 & -0.00 & 0.001 \\
\hline Earth-Moon & -0.00 & -0.00 & - & -0.00 & -0.00 & -0.00 & 0.00 & -0.00 \\
\hline Mars & 0.04 & 0.20 & -0.00 & - & 0.00 & 0.00 & -0.00 & 0.00 \\
\hline Jupiter & 5.65 & 30.37 & -0.00 & 0.28 & - & 0.34 & -0.01 & 0.03 \\
\hline Saturn & 33.94 & 182.36 & -0.001 & 1.67 & 0.17 & - & -0.05 & 0.18 \\
\hline Uranus & -0.12 & -0.67 & 0.00 & -0.01 & -0.001 & -0.01 & - & -0.001 \\
\hline Neptune & 0.02 & 0.13 & -0.00 & 0.001 & 0.00 & 0.001 & -0.00 & - \\
\hline$\omega_{j}^{(p)}$ & 39.64 & 217.61 & -0.001 & 2.00 & 0.18 & 0.40 & -0.06 & 0.22 \\
\hline
\end{tabular}

\section{References}

[1] Duboshin, G. N., Celestial Mechanics: Basic Problems and Methods, Dayton, Ohio: Foreign Technology Division, 1969.

[2] Vilke, V.G., Theoretical Mechanics, St. Petersburg: Lan', 2003 (Russian).

[3] Murray, C. D. and Dermott, S. F., Solar System Dynamics, Cambridge: Cambridge Univ. Press, 2000.

[4] Roseveare, N.T., Mercury's Perihelion from Le Verrier to Einstein, Oxford: Oxford Univ. Press, 1982.

[5] Clemence, G. M., The Relativity Effect in Planetary Motions, Rev. Mod. Phys., 1947, vol. 19, no.4, pp. 361-364.

[6] Loskutov, Yu. M., Comparison of Theoretical and Experimental Data on the Advance of the Mercury Perihelion: The Proper Contribution of the Gravitational Field of the Sun to the Perihelion Advance, Mosc. Univ. Phys. Bull., 2011, vol.66, no. 2, pp. 129-134; see also: Vestn. Mosk. Univ. Fizika, 2011, no. 2 , pp. 26-30. 